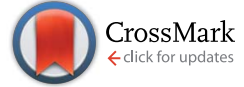

Cite this: RSC Adv., 2017, 7, 10928
Received 30th December 2016 Accepted 31st January 2017

DOI: 10.1039/c6ra28839a

rsc.li/rsc-advances

\section{Correlation between magnetic and electric properties based on the critical behavior of resistivity and percolation model of $\mathrm{La}_{0.8} \mathrm{Ba}_{0.1} \mathrm{Ca}_{0.1} \mathrm{MnO}_{3}$ polycrystalline}

\begin{abstract}
Gdaiem Mohamed Amara, ${ }^{\star a}$ Ah. Dhahri, ${ }^{\text {bc }}$ J. Dhahria and E. K. Hlil ${ }^{\text {d }}$
We investigated the magneto-electrical properties of a $\mathrm{La}_{0.8} \mathrm{Ba}_{0.1} \mathrm{Ca}_{0.1} \mathrm{MnO}_{3}$ polycrystalline sample, prepared by the polymerization-complex sol-gel method. Comparison of experimental data with the theoretical models showed that in the metal-ferromagnetic region, the electrical behavior of the sample is quite well described by a theory based on electron-electron, electron-phonon and electron-magnon scattering and Kondo-like spin dependent scattering. For the high temperature paramagnetic insulating regime, the adiabatic small polaron hopping model was found to fit well with the experimental curves. The estimated critical exponents, obtained from resistivity, were $(\beta=0.516 \pm 0.013, \gamma=1.181 \pm 0.005$ and $\alpha=0.004)$. They were very close to those predicted by the mean-field model. These results were in good agreement with the analysis of the critical exponents from magnetization measurements.
\end{abstract}

\section{Introduction}

Mixed valence manganites of a general formula $\operatorname{Tr}_{1-x} \mathrm{~A}_{x} \mathrm{MnO}_{3}$, where $\mathrm{Tr}=$ rare earth and $\mathrm{A}=\mathrm{Sr}, \mathrm{Ca}, \mathrm{Ba}$, etc. have been widely studied to understand the physics behind their properties and to explore their practical applications. ${ }^{1-3} \quad \mathrm{La}_{1-x} \mathrm{Ca}_{x} \mathrm{MnO}_{3}$ compounds have drawn much attention because of their enormous magnetoresistance (MR) and magnetocaloric effect (MCE). This makes them good candidates for a wide variety of applications. ${ }^{4-8}$ As regards the $\mathrm{La}_{1-x} \mathrm{Ca}_{x} \mathrm{MnO}_{3}$ compounds, they have a disadvantage that their Curie temperatures, $T_{\mathrm{C}}$, are well below room temperature. On the other side, doping $\mathrm{Ca}$ sites with large ions, for example $\mathrm{Pb}, \mathrm{Ba}$ and $\mathrm{Sr}$, has been explored by several researchers. ${ }^{9-11}$ The general observation is that partial doping leads to an enhancement in $T_{\mathrm{C}}$. This makes them better candidates for applications near room temperatures. The substitution of $\mathrm{Ca}$ by $\mathrm{Ba}$ is interesting as the difference between ionic sizes of the two elements is significantly large and $T_{\mathrm{C}}$ is observed to increase with increasing Ba content. ${ }^{12-14}$ Also, manganese oxides exhibit a metal-semiconductor transition (M-SC) accompanied by a ferromagnetic (FM)-paramagnetic (PM) transition near $T_{\mathrm{C}}$. The metallic behavior is usually

${ }^{a}$ Laboratoire de la Matière Condensée et des Nanosciences, Département de Physique, Faculté des Sciences de Monastir, 5019, Tunisia.E-mail: gdaiem_mohamed@hotmail.fr ${ }^{b}$ Laboratoire de Physique Appliquée, Faculté des Sciences de Sfax, Université de Sfax, BP 1171, 3000, Tunisia

${ }^{c}$ Faculté des Sciences de Monastir, Université de Monastir, Avenue de l'Environnement, Monastir-5019, Tunisia

${ }^{d}$ Institut Néel, CNRS et Université Joseph Fourier, BP 166, F-38042, Grenoble Cedex 9, France described in terms of electron scattering process and electronphonon (e-p) interaction. ${ }^{8}$ In the semiconductor region, the transport mechanism is explained by 3D Mott's variable range hopping (VRH) model, small polaron hopping (SPH) model and by the adiabatic small polaron hopping mechanism. ${ }^{15}$ Some models can explain the transport mechanism in manganites. However, most of them are only applied to fit the prominent change of the electrical resistivity curves $(\rho(T))$ in a finite temperature region (above or below $T_{\mathrm{C}}$ ). Such a model supposes that the materials are composed of PM and FM regions. Following this mechanism, $\rho(T)$ at any temperature, is determined by the change of the volume fractions of both regions. An important part of the work on manganites aimed to present the effect of MR. However, the study of the anomalies of the various thermo physical properties, like the specific heat in the vicinity of the magnetic phase transition, with investigating the values of the universal critical parameters, has not been given the sufficient importance in research projects done on manganites. As far as manganites are concerned, there have been a lot of experiments to estimate the critical exponents of FM manganites. Systems showing a second order metal insulator phase transition obey one of the common universality classes. By contrast, the experimental estimates of the critical exponents are still controversial including those for short-range Heisenberg interaction, ${ }^{\mathbf{1 6 , 1 7}}$ the mean-field values ${ }^{17,18}$ and those which cannot be classified into any universality class ever known. ${ }^{19}$

Previously, ${ }^{20}$ we have investigated in detail the structural, magnetic and MCE of our sample. In this paper, we intend to study the dependence of the electrical resistivity properties as a function of the $\mathrm{La}_{0.8} \mathrm{Ba}_{0.1} \mathrm{Ca}_{0.1} \mathrm{MnO}_{3}$, volume fraction, $f$, which 
is analyzed in the framework of the percolation theory. A percolation model based on the mixed phase consisting of itinerant electrons and localized magnetic polarons has been proposed to explain the observed results. We have also determined the critical temperature $T_{\mathrm{C}}$ and the critical parameters $\beta$ and $\gamma$. Then, we tried to investigate the critical behavior in $\mathrm{La}_{0.8} \mathrm{Ba}_{0.1} \mathrm{Ca}_{0.1} \mathrm{MnO}_{3}$ at its metal-semiconductor transition via the measurements of resistivity. The values of these exponents obtained from resistivity are very close to those predicted by the mean-field model.

\section{Experimental details}

The nano-polycrystalline sample of a nominal composition $\mathrm{La}_{0.8} \mathrm{Ba}_{0.1} \mathrm{Ca}_{0.1} \mathrm{MnO}_{3}$ was prepared according to the polymerization complex sol-gel method. The detailed basic physical properties are reported in ref. 20. The crystal structure of the final product was checked by X-ray diffraction, which confirmed that the sample having a single phase, crystallized in the rhombohedral structure with $R \overline{3} c$ space group. ${ }^{20}$ In the present study the electrical transport properties $\rho(T)$ were measured by a standard four-probe method using physical property measurement system (PPMS, Quantum Design).

\section{Results and discussions}

\subsection{Electrical properties}

First, we discuss electrical resistivity results at an applied magnetic field of 0 to $5 \mathrm{~T}$, with steps of $0.5 \mathrm{~T}$. This is shown in Fig. 1. Taking the sign of the temperature derivative of $\mathrm{d} \rho / \mathrm{d} T$ as a criterion, we found that samples exhibit a metallic behavior $\mathrm{d} \rho / \mathrm{d} T>0$ at a low temperature $\left(T<T_{\mathrm{M}-\mathrm{SC}}\right)$ and become semiconductor like $\mathrm{d} \rho / \mathrm{d} T$ above the temperature $T_{\mathrm{M}-\mathrm{SC}}$, where $T_{\mathrm{M}-\mathrm{SC}}$ is the temperature of the maximum value of resistivity. The transition temperature $T_{\mathrm{M}-\mathrm{SC}}$ occurs at $T_{\mathrm{M}-\mathrm{SC}}=303 \mathrm{~K}$, which is close to its $T_{\mathrm{C}}=282 \mathrm{~K},{ }^{20}$ indicating strong correlations between the magnetic and electrical properties in our sample. Therefore, we can define the FM-metallic-like and the PM-semiconductorlike behaviors as a function of temperature. It is also clearly seen that in the vicinity of $T_{\mathrm{M} \text {-SC }}$, resistivity decreases with the increase of the applied magnetic field. The values of $T_{\mathrm{M} \text {-SC }}$ are found to move toward a high temperature side with increasing the magnetic field. The observed behavior may be attributed to the fact that the applied magnetic field suppresses the electrical resistivity by several orders. This is attributed to the induced magnetic ordering of the localized $t_{2 g}$ spins and delocalization of charge carriers on the application of magnetic field..$^{21}$ Due to this ordering, the FM metallic state may suppress the magnetic insulating regime. We can note that the electrical properties of manganites are generally understood according to the double exchange theory (DE). ${ }^{22}$ In this model, the $\mathrm{Mn}^{3+}-\mathrm{O}-\mathrm{Mn}^{4+}$ coupling is responsible of the conduction mechanisms from the half-filled to the empty $e_{g}$ orbital. As a result, the conduction electrons are completely polarized inside the magnetic domains and are easily transferred between the pairs $\mathrm{Mn}^{3+}\left(3 \mathrm{~d}^{4}\right.$, $\left.\mathrm{t}_{2 \mathrm{~g}}^{3} \uparrow \mathrm{e}_{\mathrm{g}}^{1} \uparrow, S=2\right)$ and $\mathrm{Mn}^{4+}\left(3 \mathrm{~d}^{3}, \mathrm{t}_{2 \mathrm{~g}}^{3} \uparrow \mathrm{e}_{\mathrm{g}}^{0}, S=3 / 2\right)$ via oxygen.

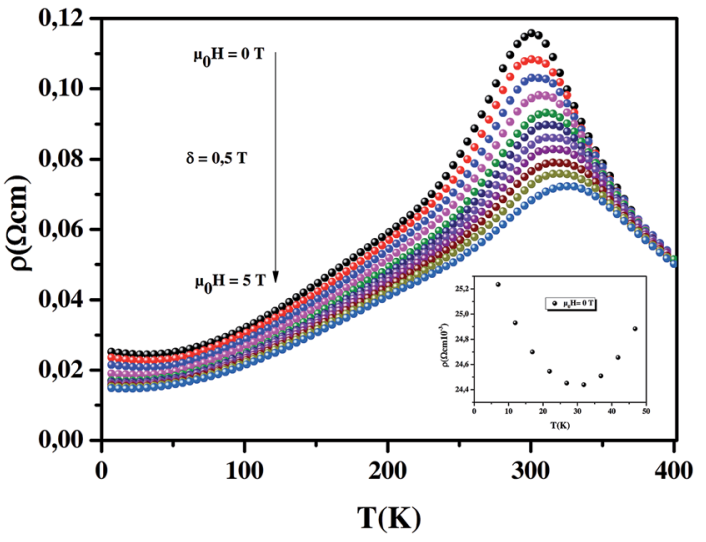

Fig. 1 Temperature dependence of the resistivity under different applied magnetic fields from 0 to $5 \mathrm{~T}$, with steps of $0.5 \mathrm{~T}$. The inset shows temperature dependence of electrical resistivity at very low temperature for our sample.

In what follows, we analyze the electrical resistivity data using different theoretical models in order to understand the contribution of different parameters to the conduction mechanism in different temperature regimes. The quality of fitting was judged by the squared linear correlation coefficients $\left(R^{2}\right)$. It is important to mention that the obtained values of $R^{2}$ for our sample are high value.

3.1.1. $\boldsymbol{T}<\boldsymbol{T}_{\mathbf{M}-\mathrm{SC}}$ region. In the low-temperature, the transport properties fully show the metallic state by fitting with the following equation, which is generally used to fit the electrical resistivity data in the case of manganites: $:^{23,24}$

$$
\rho(T)=\rho_{0}+\rho_{2} T^{2}+\rho_{4.5} T^{4.5}
$$

where $\rho_{0}$ is the residual resistivity arising from the temperature independent processes such as domain wall, grain boundary and vacancies, $\rho_{2} T^{2}$ (ref. 25,26) indicates electron-electron (e-e) scattering, ${ }^{27}$ whereas $\rho_{4.5} T^{4.5}$ is a combination of e-e, electronmagnon (e-m) and $\mathrm{e}-\mathrm{p}$ scattering processes. ${ }^{28,29}$ However, as this model is not in a position to explain the low temperature

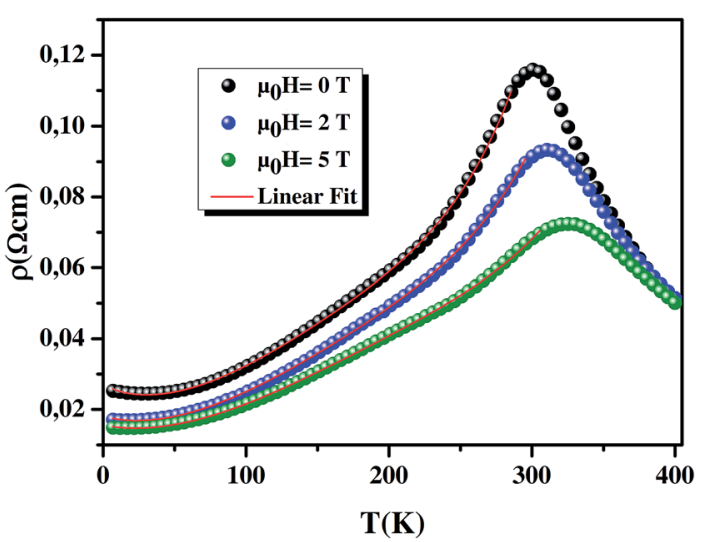

Fig. 2 The plots of the electrical resistivity $\rho(T)$ vs. T at 0, 2 and $5 T$. The red solid line in these plots represents the best fit of experimental data in the metallic regime below $\left(T_{M-s C}\right)$ with eqn (2). 
Table 1 The best fit parameters obtained from the experimental resistivity data of the metallic behavior (below $T_{M-s c)}$

\begin{tabular}{llllll}
\hline$\mu_{0} H(T)$ & 0 & 1 & 2 & 3 & 5 \\
\hline$\rho_{0}(\Omega \mathrm{cm})$ & 0.02854 & 0.02442 & 0.01891 & 0.01741 & 0.01689 \\
$\rho_{1 / 2}\left(10^{-4} \Omega \mathrm{cm} \mathrm{K}^{-1 / 2}\right)$ & 10.70100 & 9.46491 & 5.71586 & 4.73059 & 4.59980 \\
$\rho_{1 / 2}\left(10^{-7} \Omega \mathrm{cm} \mathrm{K}^{-2}\right)$ & 1.62043 & 1.52213 & 1.29249 & 1.18636 & 1.12878 \\
$\rho_{4.5}\left(10^{-13} \Omega \mathrm{cm} \mathrm{K}^{-4.5}\right)$ & -3.72372 & -3.24886 & -2.31117 & -1.98137 & -1.81468 \\
$R^{2}(\times 100)$ & 99.899 & 99.954 & 99.914 & 99.887 & 9.52850 \\
& & & & & 99.901 \\
\hline
\end{tabular}

upturn in resistivity (seen in the inset of Fig. 1), we concluded that along with the above mentioned phenomena, some other factors might have also contributed to the low temperature behavior. $^{30}$

Based on the strong correlated effect in manganites, one has to consider the e-e interaction, which causes a $T^{1 / 2}$ dependence of resistivity in a disordered system. ${ }^{31}$

Therefore, in order to explain the origin of the low temperature resistivity upturn, the experimental data were analyzed by taking into account the Kondo-like scattering, e-e interaction and $\mathrm{e}-\mathrm{p}$ interaction. To represent these phenomena, two more terms were included in eqn (1) and the new equation is:

$$
\rho(T)=\rho_{0}-\rho_{1 / 2} T^{1 / 2}+\rho_{2} T^{2}+\rho_{4.5} T^{4.5}+\rho_{5} T^{5}
$$

where $\rho_{1 / 2} T^{1 / 2}$ is in relation with e-e coulombic interactions and $\rho_{5} T^{5}$ is due to e-p interactions. Plots of the best fits to the data are shown by red solid lines in the main panels of Fig. 2 . The best fitting parameters are listed in Table 1.

3.1.2. $\boldsymbol{T}>\boldsymbol{T}_{\mathbf{M} \text {-SC }}$ region. The electrical behavior of our sample, in the semiconductor region can explained using two different models viz., SPH model and VRH model. ${ }^{32}$ The high temperature region above $\theta_{\mathrm{D}} / 2$ (where $\theta_{\mathrm{D}}$ is Debye's temperature $^{33}$ ) is analyzed using the SPH model, whereas below $\theta_{\mathrm{D}} / 2$ and above $T_{\mathrm{M}-\mathrm{SC}}$, it is analyzed using VRH model.

Firstly, we discuss the SPH model which is given by the equation: ${ }^{34}$

$$
\rho(T)=A^{\prime} T \exp \left(\frac{E_{\mathrm{a}}}{k_{\mathrm{B}} T}\right)
$$

where $A^{\prime}=\left[k_{\mathrm{B}} / n_{\mathrm{ph}} N e^{2} R^{2} C(1-C) \exp (2 a R)\right], k_{\mathrm{B}}$ is Boltzmann's constant, $N$ is the number of ion sites per unit volume, $R$ is the average intersite spacing obtained from the relation $R=(1 / N)^{1 /}$ ${ }^{3}, C$ is the fraction of sites occupied by the polaron, a is the electron wave function decay constant, $n_{\mathrm{ph}}$ is the optical phonon frequency $\left(n_{\mathrm{ph}}=k_{\mathrm{B}} \theta_{\mathrm{D}} / h\right), T$ is the absolute temperature and $E_{\mathrm{a}}$ is the activation energy. The temperature dependent polaron hopping, verified by calculating $\theta_{\mathrm{D}} / 2$, presents the deviation from linearity which occurs in the $\ln (\rho / T) v s . T^{-1}$ plot. For the validity of this model, a plot of $\ln (\rho / T) v s . T^{-1}$ is expected to be a straight line, shown in Fig. 3(a). From the interception and the slope of the linear curve above $\theta_{\mathrm{D}} / 2, A^{\prime}$ and $E_{\mathrm{a}}$ are estimated, respectively, using eqn (3) (Table 2). The value of $A^{\prime}$ increases with the increase in the magnetic field, which facilitates the hopping of the electrons to the nearby neighboring states. Also, we can see that $\theta_{\mathrm{D}}$ increases, whereas $E_{\mathrm{a}}$ decreases gradually as the magnetic field increases. This can be explained on the basis of the delocalization of $e_{g}$ electrons due to the application of magnetic field. ${ }^{35}$

To verify the conduction mechanism in the low temperature region $\left(T<\theta_{\mathrm{D} / 2}\right)$, we fitted the temperature dependence of electrical resistivity using VRH model. The VRH model is applicable when the thermal energy is insufficient to excite the electrons to hop to their nearest neighbors. It is more favorable for the electrons to hop further to find a site with a smaller potential difference. The VRH model for three dimensional systems is given by: ${ }^{27}$

$$
\rho=\rho_{0} \exp \left(T_{0} / T\right)^{1 / 4}
$$

where $T_{0}$ is the characteristic temperature which is expressed as $T_{0}=\left(16 \alpha^{3} / k_{\mathrm{B}} N\left(E_{\mathrm{F}}\right)\right), \alpha$ which is the inverse of the localization length of the trapped charge carriers, was taken as $2.22 \mathrm{~nm}^{-1},{ }^{27}$ $N\left(E_{\mathrm{F}}\right)$ is the density of state at Fermi level and $\rho_{0}$ is the residual
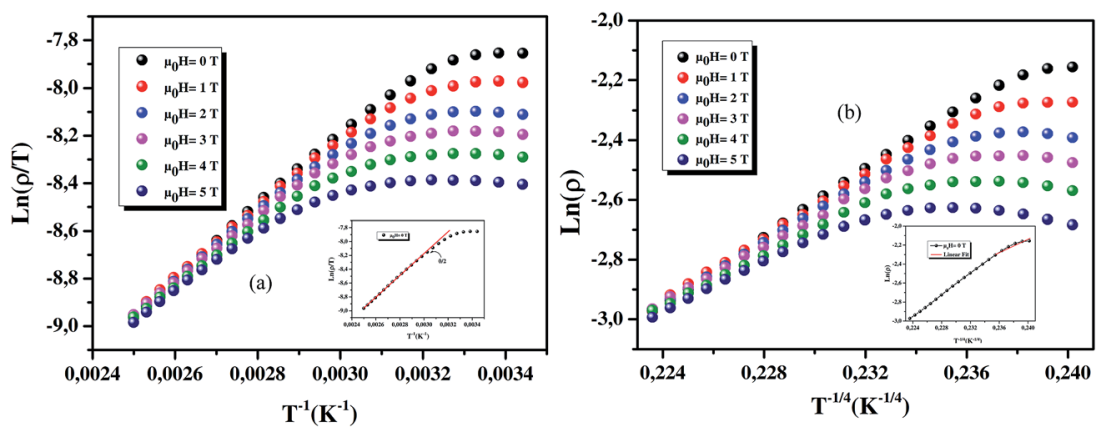

Fig. 3 (a) Plot $\ln (\rho / T)$ versus $\left(T^{-1}\right)$ for sample in the presence and the absence of the magnetic field. Solid lines are the best fit to eqn (3). The inset of (a) shows as an example of the $\ln (\rho / T)$ versus $\left(T^{-1}\right)$ at $0 \mathrm{~T}$. (b) Plot $\ln (\rho)$ versus $\left(T^{-1 / 4}\right)$ for sample in the presence and the absence of the magnetic field. Solid lines are the best fit to eqn (4). The inset of (b) shows as an example of the $\ln (\rho)$ versus $\left(T^{-1 / 4}\right)$ at $0 \mathrm{~T}$. 
Table 2 The best fit parameters obtained from the experimental resistivity data for semi-conductor behavior (above $T_{M}$-sc)

\begin{tabular}{llllll}
\hline$\mu_{0} H(T)$ & 0 & 1 & 2 & 3 & 4 \\
\hline$\theta_{\mathrm{D}} / 2$ & 650.813 & 660.767 & 670.734 & 680.765 & 690.661 \\
SPH & & & & 710.576 \\
$A^{\prime}\left(10^{-6} \Omega \mathrm{cm}\right)$ & 2.73707 & 3.34612 & 3.373235 & 4.33607 & 5.06174 \\
$E_{\mathrm{a}} / k_{\mathrm{B}}(\mathrm{K})$ & 1541.19162 & 1456.73030 & 1420.23404 & 1361.11196 & 1292.74212 \\
$R^{2}(\times 100)$ & 99.838 & 99.974 & 99.954 & 99.883 & 99.931 \\
VRH & & & & 9.37541 \\
$R^{2}(\times 100)$ & 91.721 & 80.962 & 56.680 & 40.237 & 1262.75730 \\
\hline
\end{tabular}

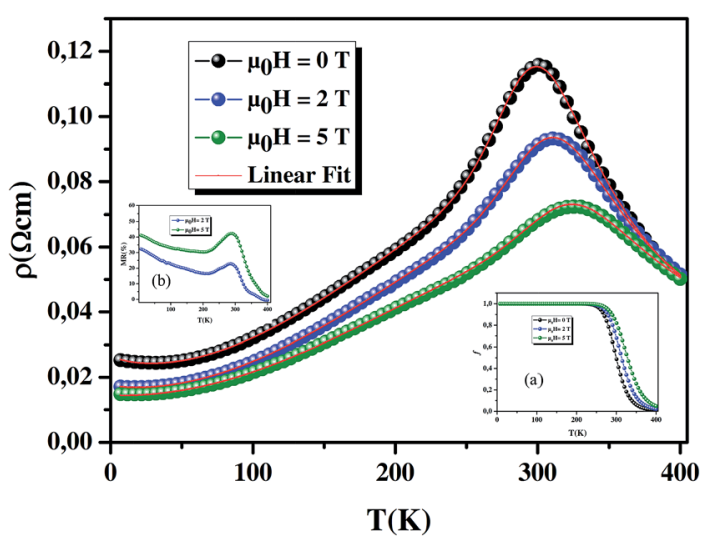

Fig. 4 The temperature dependence of resistivity under various magnetic fields of 0,2 and $5 \mathrm{~T}$, as an example. Symbols are the experimental data and solid lines are the resistivity calculated using eqn (10). The inset (a) shows the temperature dependence of FM phase volume fraction $f$ for our sample under applied magnetic field of 2 and $5 \mathrm{~T}$. The inset (b) shows the variation of MR vs. T curves.

resistivity. Here, Fig. 3(b) shows $\ln (\rho)$ versus $\left(T^{-1 / 4}\right)$ for our sample in the presence and the absence of the magnetic field. Solid lines are the best fit to eqn (4). The fitting parameters in this model are listed in Table 2. We can see weak values of the square linear correlation coefficients $\left(R^{2}\right)$. This is due to the invalidity of VRH model, to describe the $T_{\mathrm{M}-\mathrm{SC}}<T<\theta_{\mathrm{D} / 2}$ region.

Both models describe quite well our results in the semiconductor behavior, although the SPH model gives the best square linear correlation coefficients $\left(R^{2}\right)$. We may conclude then, that the transport properties are dominated by the SPH mechanism.

\subsection{Percolation model}

None of the mentioned models can explain the significant change in $\rho$ vs. $T$ curves near $T_{\mathrm{M}-\mathrm{Sc}}$. Later, Goodenough ${ }^{36}$ showed that in CMR materials, a metallic conductivity exists in the FM regions and a conductivity semiconductor at above $T_{\mathrm{M}-}$ sc in the PM regions. Recently, based on the phase segregation mechanism Li et al. ${ }^{37}$ have suggested that the FM and PM group co-exist in MR regions (percolation model) and at any temperature, $\rho$ is determined by the variation volume fractions of the two regions. Under this scenario, a complete expression for resistivity can be written as follows: ${ }^{37}$

$$
\rho=\rho_{\mathrm{FM}} f+(1-f) \rho_{\mathrm{PM}}
$$

where $f$ and $(1-f)$ are the volume concentrations of the FM domains and PM regions, respectively. The volume concentrations of FM and PM phases satisfy Boltzman's distribution:

$$
f=\frac{1}{1+\exp \left(\frac{\Delta U}{k_{\mathrm{B}} T}\right)}
$$

and

$$
1-f=\frac{\exp \left(\frac{\Delta U}{k_{\mathrm{B}} T}\right)}{1+\exp \left(\frac{\Delta U}{k_{\mathrm{B}} T}\right)}
$$

where $\Delta U$ is the energy difference between FM and PM states, it may be expressed as:

$$
\Delta U \approx-U_{0}\left(1-\frac{T}{T_{\mathrm{C}}^{\bmod }}\right)
$$

$T_{\mathrm{C}}^{\mathrm{mod}}$ means a temperature in the vicinity of which the resistivity reaches a maximum value. ${ }^{38} U_{0}$ is taken as the energy difference for temperatures well below $T_{\mathrm{C}}^{\bmod } .^{37}$

From eqns (6)-(8), one can find that:

$$
\begin{aligned}
f & =0 \text { for } T \gg T_{\mathrm{C}}^{\bmod }, f=1 \text { for } T \ll T_{\mathrm{C}}^{\bmod } \text { and } \\
f & =f_{\mathrm{c}}=\frac{1}{2} \text { at } T=T_{\mathrm{C}}^{\bmod } \cdot 37,38 \\
1-f & =1 \text { for } T \gg T_{\mathrm{C}}^{\bmod }, 1-f=0 \text { for } T \ll T_{\mathrm{C}}^{\bmod } \text { and } \\
1-f & =f_{\mathrm{c}}=\frac{1}{2} \text { at } T=T_{\mathrm{C}}^{\bmod } \cdot 37,38
\end{aligned}
$$

where $f_{\mathrm{C}}$ is the percolation threshold. To conclude, when $f$ is less than $f_{\mathrm{C}}$, the sample remains semiconducting and when $f$ is larger than $f_{\mathrm{C}}$, it becomes metallic. Hence the complete expression describing the resistivity dependence on the temperature can be written in the form:

$$
\begin{aligned}
\rho(T)= & \rho_{0}-\rho_{1 / 2} T^{1 / 2}+\rho_{2} T^{2}+\rho_{4.5} T^{4.5}+\rho_{5} T^{5} f \\
& +A^{\prime} T \exp \left(\frac{E_{\mathrm{a}}}{k_{\mathrm{B}} T}\right)(1-f)
\end{aligned}
$$


Table 3 Obtained parameters corresponding to the best fit to eqn (9) of the experimental data of our sample at 0 to $5 \mathrm{~T}$, with steps of $1 \mathrm{~T}$

\begin{tabular}{llllll}
\hline$U_{0} H(T)$ & 0 & 1 & 2 & 3 & 5 \\
\hline$\rho_{0}(\Omega \mathrm{cm})$ & 0.02755 & 0.02309 & 0.01793 & 0.01635 & 0.01543 \\
$\rho_{1 / 2}\left(10^{-4} \Omega \mathrm{cm} \mathrm{K}^{0.5}\right)$ & 8.11099 & 6.14486 & 3.28384 & 1.66104 & 1.30253 \\
$\rho_{2}\left(10^{-7} \Omega \mathrm{cm} \mathrm{K}^{-2}\right)$ & 13.239 & 11.9077 & 10.5331 & 9.45765 & 8.85296 \\
$\rho_{4.5}\left(10^{-13} \Omega \mathrm{cm} \mathrm{K}^{-4.5}\right)$ & -9.40284 & -6.09755 & -4.64322 & -3.98003 & -3.62861 \\
$\rho_{5}\left(10^{-15} \Omega \mathrm{cm} \mathrm{K}^{-5}\right)$ & 35.6269 & 18.6148 & 13.1506 & 11.2931 & 9.01469 \\
$\rho_{0}\left(10^{-6} \Omega \mathrm{cm}\right)$ & 2.35031 & 2.91272 & 3.17748 & 3.64326 & 4.21711 \\
$E_{\mathrm{a}} / k_{\mathrm{B}}(\mathrm{K})$ & 1592.62715 & 1515.48663 & 1476.0954 & 1420.12229 & 1363.35483 \\
$U_{0} / k_{\mathrm{B}}(\mathrm{K})$ & 5667.05496 & 5601.49997 & 5565.38411 & 5522.66875 & 5475.50923 \\
$R^{2}(\times 100)$ & 99.838 & 99.974 & 99.954 & 99.883 & 9.1674 \\
\end{tabular}

The total $(\rho)$ can be represented as:

$$
\begin{aligned}
\rho(T)= & \rho_{0}-\rho_{\frac{1}{2}} T^{\frac{1}{2}}+\rho_{2} T^{2}+\rho_{4.5} T^{4.5}+\rho_{5} T^{5} \\
& \times \frac{1}{1+\exp \left(\frac{-U_{0}\left(1-\frac{T}{T_{\mathrm{C}}^{\mathrm{mod}}}\right)}{k_{\mathrm{B}} T}\right)}+A^{\prime} T \exp \left(\frac{E_{\mathrm{a}}}{k_{\mathrm{B}} T}\right) \\
& \times \frac{\exp \left(\frac{-U_{0}\left(1-\frac{T}{T_{\mathrm{C}}^{\mathrm{mod}}}\right)}{k_{\mathrm{B}} T}\right)}{\left(\frac{-U_{0}\left(1-\frac{T}{T_{\mathrm{C}}^{\mathrm{mod}}}\right)}{k_{\mathrm{B}} T}\right)} \\
& 1+\exp \left(\frac{1}{}\right)
\end{aligned}
$$

Fig. 4 shows the simulated and the experimental results for the $\rho(T)$ curve obtained in zero field and with an applied magnetic field $2 \mathrm{~T}$ and $5 \mathrm{~T}$ for $\mathrm{La}_{0.8} \mathrm{Ba}_{0.1} \mathrm{Ca}_{0.1} \mathrm{MnO}_{3}$. The best fit parameters are listed in Table 3. It is worth mentioning that this model is suitable to explain the electrical transport of $\mathrm{La}_{0.8^{-}}$ $\mathrm{Ba}_{0.1} \mathrm{Ca}_{0.1} \mathrm{MnO}_{3}$. From Table 3 , the reduction in $\rho_{2}$ and $\rho_{9 / 2}$ could be attributed to the decrease in electron spin fluctuations in the presence of a magnetic field. One also can notice the decrease of $E_{\mathrm{a}}$ and $U_{0}$ with increasing the magnetic field. This is due to the spin attempt to align along the magnetic field, which favors the conduction and decrease the ability of charge localization. Morever, the electrons jumping requires less energy. This implies that $e_{g}$ electron hopping becomes easier and needs less energy. $T_{\mathrm{C}}^{\text {mod }}$ shows an increasing behavior as a function of the applied field due to the enhancement of metallic tendencies which shifts $T_{\mathrm{M}-\mathrm{SC}}$ to higher values and so $T_{\mathrm{C}}^{\mathrm{mod}}$ from 299.661 to $327.863 \mathrm{~K}$, respectively at an applied magnetic field of 0 and $5 \mathrm{~T}$.

The inset (a) of Fig. 4 shows the temperature dependence of the volume fraction of the FM phase $f$ in a zero magnetic field and in an applied magnetic field of $2 \mathrm{~T}$ and $5 \mathrm{~T}$. When the temperature is considerably below $T_{\mathrm{C}}, f$ is close to 1 , which means that the sample is completely FM. By increasing the
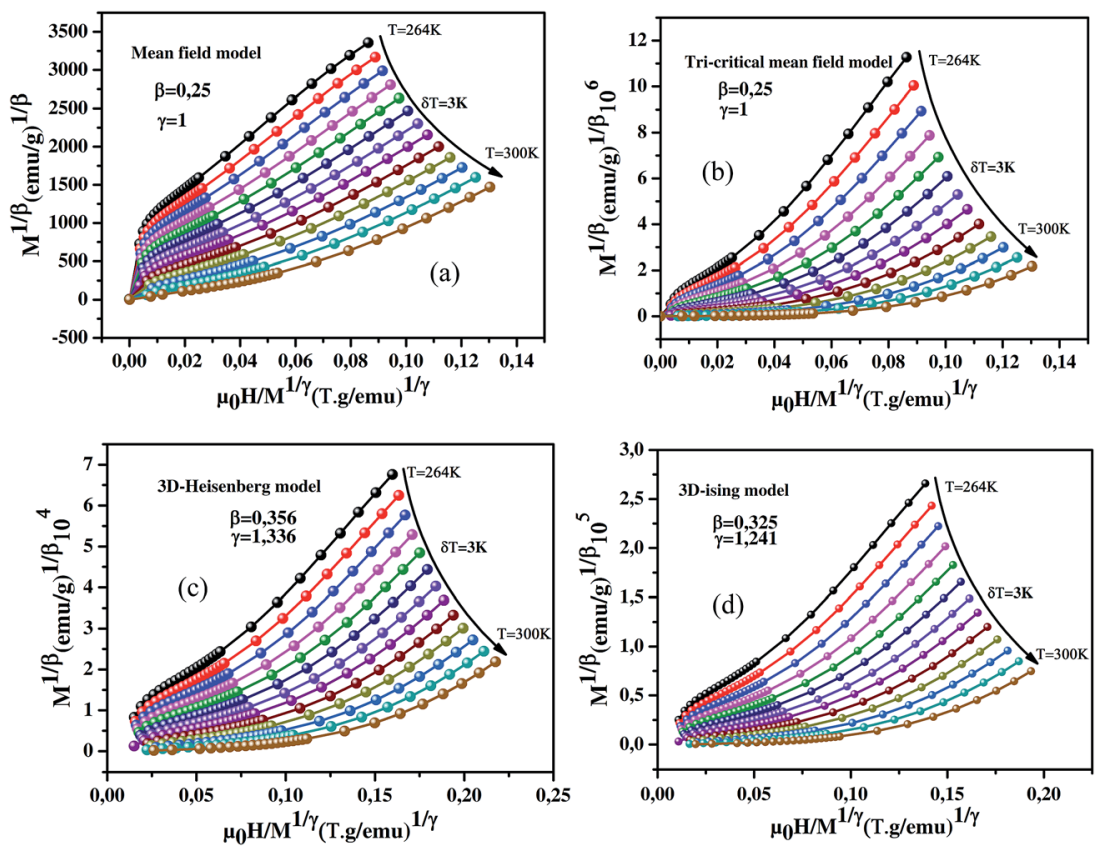

Fig. 5 MAP for our sample: isotherms of $M^{1 / \beta}$ vs. $\left(\mu_{0} H / M\right)^{1 / \gamma}$; (a) mean field model $(\beta=0.25, \gamma=1)$, (b) tri-critical mean-field model $(\beta=0.25, \gamma=$ 1), (c) 3D-Heisenberg model $(\beta=0.365, \gamma=1.336)$ and (d) 3D-Ising model $(\beta=0.325, \gamma=1.24)$. 


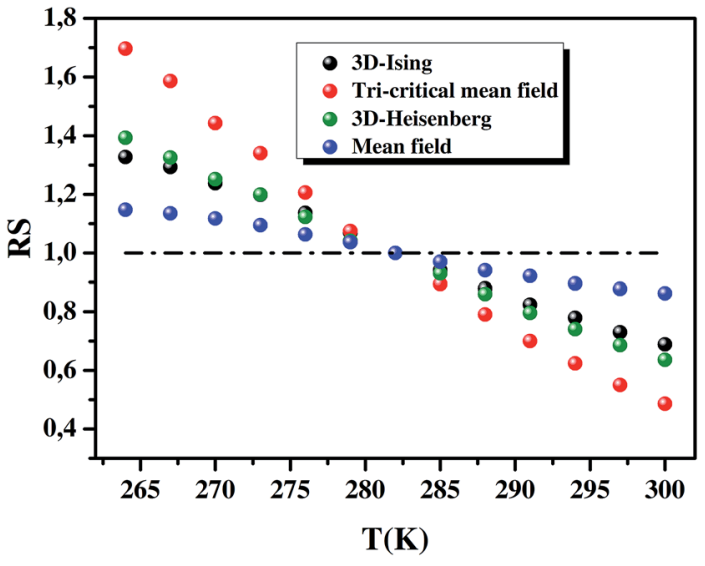

Fig. 6 RS of our sample as a function of temperature defined as RS = $S(T) / S\left(T_{C}\right)$, using several methods.

temperature, $f$ decreases slowly and reaches the percolation threshold $f_{\mathrm{C}}=0.5$ at $T_{\mathrm{C}}^{\mathrm{mod}}$. In the high temperature region, the value of $f$ becomes equal to zero, which means that the sample is completely PM. Increasing the applied magnetic field value will certainly enhance $f$ value due to the enhancement of FM metallic interactions attributed to zener DE mechanism.

\subsection{Study of magnetoresistance}

The coexistence of ferromagnetism and metallic conductivity causes the relatively strong effect of magnetoresistance (MR). The MR $(T)$ at a given temperature is defined as:

$$
\operatorname{MR} \%=\frac{\left[\rho(0, T)-\rho\left(\mu_{0} H, T\right)\right]}{\rho(0, \mathrm{~T})}
$$

where $\rho(0)$ is the resistivity under a zero magnetic field and $\rho\left(\mu_{0} H\right)$ is the resistivity under an applied field of 2 and $5 \mathrm{~T}$.

The MR dependence on the temperature is shown in the inset (b) of Fig. 4 for the studied sample at different applied magnetic fields ( 2 and $5 \mathrm{~T}$ ), over a temperature range of $4-400 \mathrm{~K}$. We can see that our results could even be better that those found in ref. 39-43.

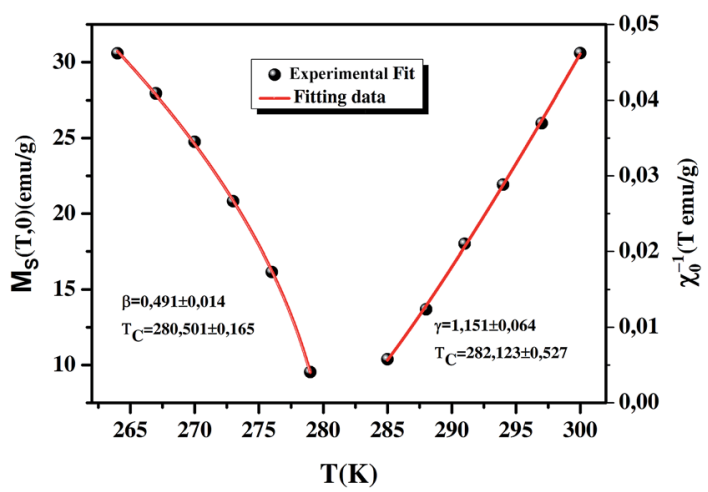

Fig. 7 Temperature dependence of the spontaneous magnetization $M_{S}(T, 0)$ (left) and the inverse initial susceptibility $\chi_{0}{ }^{-1}(T)$ (right), with the fitting curves based on the power laws.

\subsection{Critical parameters}

3.4.1. Critical parameters determined from magnetization data. To analyze the nature of the magnetic transition in $\mathrm{La}_{0.8^{-}}$ $\mathrm{Ba}_{0.1} \mathrm{Ca}_{0.1} \mathrm{MnO}_{3}$ sample, the critical behavior near $T_{\mathrm{C}}$ is analysed. According to the scaling hypothesis, a continuous phase transition near the critical temperature $T_{\mathrm{C}}$ shows a power law dependence of spontaneous magnetization $\left(M_{\mathrm{S}}(T)\right)$, and inverse initial susceptibility $\left(\chi_{0}{ }^{-1}(T)\right)$ on the reduced temperature with a set of interdependent critical exponents $\beta, \gamma$ and $\delta$. The mathematical definitions of the critical exponents from magnetization measurements are given by the following relations: ${ }^{44-47}$

$$
\begin{gathered}
M_{\mathrm{S}}(T)=M_{0}|\varepsilon|^{-\beta}, \varepsilon<0, T<T_{\mathrm{C}} \\
\chi_{0}^{-1}(T)=\left(h_{0} / M_{0}\right) \varepsilon^{\gamma}, \varepsilon>0, T>T_{\mathrm{C}} \\
M=D H^{1 / \delta}, \varepsilon=0, T=T_{\mathrm{C}}
\end{gathered}
$$

where $\beta$ is the spontaneous magnetization exponent, $\gamma$ is the isothermal magnetic susceptibility exponent, $\delta$ is the critical isotherm exponent, $M_{0}$ and $h_{0}$ are the critical amplitudes and $\varepsilon$ $=\left(T-T_{\mathrm{C}}\right) / T_{\mathrm{C}}$ is the reduced temperature.

Therefore, to obtain the right values of $\beta$ and $\gamma$ exponents, we have included the analysis of the critical exponents using a modified Arrott plot (MAP) expression. This method was based on the Arrott-Noakes equation of state: ${ }^{48}$

$$
\left(\mu_{0} H / M\right)^{1 / \gamma}=a\left(T-T_{\mathrm{C}}\right) / T+b M^{1 / \beta}
$$

where $a$ and $b$ are considered to be constants in the mean-field theory, values of $\beta=0.5$ and $\gamma=1$ should generate the regular Arrott's plots, $M^{2}$ vs. $\mu_{0} H / M$.

The $(M)^{1 / \beta}$ vs. $\left(\mu_{0} H / M\right)^{1 / \gamma}$ Arrott-Noakes plots are constructed for our compound using different kinds of trial exponents. The curves are displayed in Fig. 5(a-d) mean-field model $(\beta=0.5, \gamma$ $=1)$, tricritical mean-field model $(\beta=0.25, \gamma=1)$, 3DHeisenberg model $(\beta=0.365, \gamma=1.336)$ and 3D-Ising model ( $\beta=0.325, \gamma=1.241)$, respectively. To distinguish which model better describes this system, we calculated the so-called relative slope (RS) defined at the critical point: $\mathrm{RS}=S(T) / S\left(T_{\mathrm{C}}\right)$. The $S(T)$ and $S\left(T_{\mathrm{C}}\right)$ are the slope for a given $T$ close to $T_{\mathrm{C}}$ and the slope at

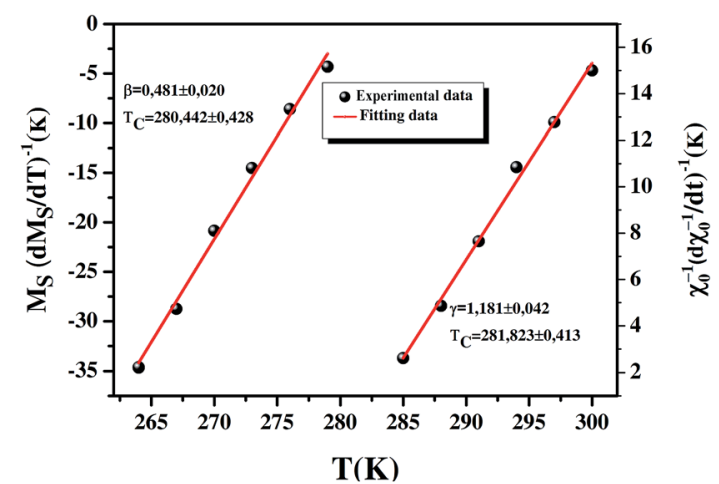

Fig. $8 \mathrm{KF}$ plots for the spontaneous magnetization (left) and the inverse initial susceptibility $\chi_{0}{ }^{-1}(T)$ (right). 


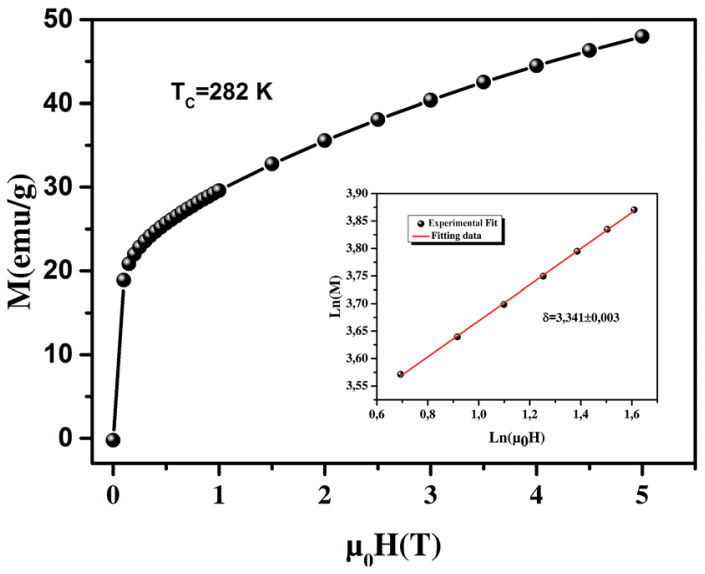

Fig. 9 Isothermal $M\left(T_{C}, \mu_{0} H\right)$ plot for our sample at $T_{C}=282$. The inset shows the same plot in $\ln -\ln$ scale and the solid line is the linear fit following eqn (14), for the determination of the critical exponent $\delta$.

$T=T_{\mathrm{C}}$, successively. The considered $T_{\mathrm{C}}=282 \mathrm{~K}$ is determined from the Arrott-Nokaes curves, where at $T=282 \mathrm{~K}$, the curves $M^{1 / \beta} v s .\left(\mu_{0} H / M\right)^{1 / \gamma}$ for our model is a linear curve crossing the origin. If the MAP shows a series of absolute parallel lines, the relative slope of the most satisfactory model should be kept to 1 irrespective of temperatures. ${ }^{49} \mathrm{RS} v s$. $T$ curves for all models are shown in Fig. 6. One can see that the mean field model is the best one for the determination of the critical exponents for our sample. Following a standard procedure, the spontaneous magnetization $v s$. temperature, would be obtained from the linear extrapolation in the high magnetic field region for the isotherms to the coordinate axis of $M^{1 / \beta}$. Similarly, $\chi_{0}{ }^{-1}(T)$, is also obtained from the intersections with the $\left(\mu_{0} H / M\right)^{1 / \gamma}$ axis. By fitting the $M_{\mathrm{S}}(T)$ and $\chi_{0}{ }^{-1}(T)$ data to eqns (12) and (13), respectively, new values of $\beta$ and $\gamma$ will be obtained. These values are then re-introduced to the scaling of the MAP. After several times of such scaling, $\beta$ and $\gamma$ converge to their optimal values. Concurrently, the Curie temperatures associated with the fitting of the $M_{\mathrm{S}}(T)$ and $\chi_{0}{ }^{-1}(T)$ data to eqns (12) and (13), respectively, are also determined $M_{\mathrm{S}} v s . T$ and $\chi_{0}{ }^{-1}(T) v s . T$ are plotted in Fig. 7. Eqn (12) gives the value of $\beta=0.491 \pm 0.014$

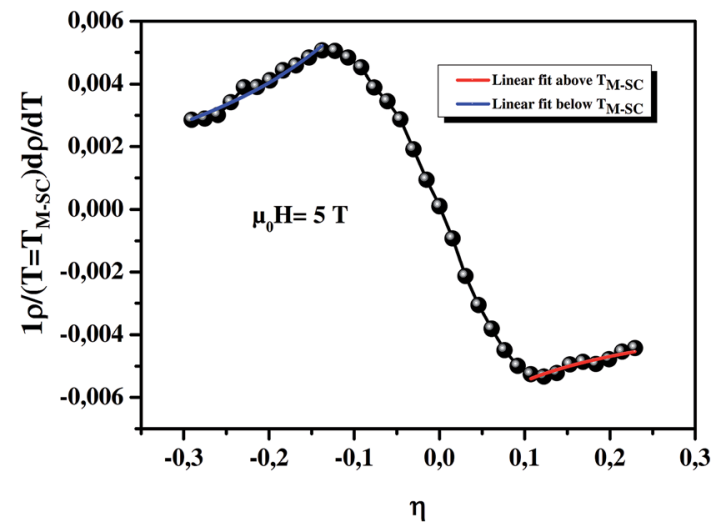

Fig. 10 Thermal derivative of resistivity normalized with respect to resistivity value at $T_{M-S C}$ as a function of reduced temperature, $\eta$.

with $T_{\mathrm{C}}=280.501 \pm 0.165 \mathrm{~K}$ and eqn (13) gives the value of $\gamma=$ $1.151 \pm 0.064$ with $T_{\mathrm{C}}=282.123 \pm 0.527 \mathrm{~K}$.

Such critical exponents and $T_{\mathrm{C}}$ can be obtained more accurately by analyzing the $M_{\mathrm{S}}(T)$ and $\chi_{0}{ }^{-1}(T)$ with the Kouvel-Fisher (KF) method: $:^{50,51}$

$$
\begin{gathered}
M_{\mathrm{S}}(T) /\left[\mathrm{d} M_{\mathrm{S}}(T) / \mathrm{d} T\right]=\left(T-T_{\mathrm{C}}\right) / \beta \\
\chi_{0}^{-1}(T) /\left[\mathrm{d} \chi_{0}^{-1}(T) / \mathrm{d} T\right]=\left(T-T_{\mathrm{C}}\right) / \gamma
\end{gathered}
$$

The plots of $M_{\mathrm{S}}(T) /\left[\mathrm{d} M_{\mathrm{S}}(T) / \mathrm{d} T\right]$ vs. $T$ and $\chi_{0}{ }^{-1}(T) /\left[\mathrm{d} \chi_{0}{ }^{-1}(T) /\right.$ $\mathrm{d} T] v s$. $T$ should yield straight lines with slopes of $1 / \beta$ and $1 / \gamma$, respectively, and the intercepts on the axes are equal to $T_{\mathrm{C}}$. The linear fitting to the plots following the KF method gives $\beta=$ $0.481 \pm 0.020$ with $T_{\mathrm{C}}=280.442 \pm 0.428 \mathrm{~K}$ and $\gamma=1.181 \pm$ 0.042 with $T_{\mathrm{C}}=281.823 \pm 0.413 \mathrm{~K}$ (Fig. 8). Obviously, the obtained values of the critical exponents and $T_{\mathrm{C}}$, using the $\mathrm{KF}$ method, are in agreement with that using the MAP of the meanfield model. Concerning the value of $\delta$, it can be directly obtained from plotting the critical isotherm at $T_{\mathrm{C}}$. In Fig. 9, the $M$ vs. $\mu_{0} H$ curve at $282 \mathrm{~K}$ was chosen as the critical isotherm based on the previous discussion. The inset of Fig. 9 shows the same curve $M v s$. $\mu_{0} H$ on a ln-ln scale. The high-field region of the

\begin{tabular}{|c|c|c|c|c|c|c|}
\hline System & Method & $T_{\mathrm{C}}(\mathrm{K})$ & $\beta$ & $\gamma$ & $\delta$ & Ref. \\
\hline $\mathrm{La}_{0.8} \mathrm{Ba}_{0.1} \mathrm{Ca}_{0.1} \mathrm{MnO}_{3}$ & MAP & $281.312 \pm 0.144$ & $0.491 \pm 0.014$ & $1.151 \pm 0.064$ & 3.344 & This work \\
\hline $\mathrm{La}_{0.8} \mathrm{Ba}_{0.1} \mathrm{Ca}_{0.1} \mathrm{MnO}_{3}$ & $\mathrm{KF}$ & $381.133 \pm 0.42$ & $0.481 \pm 0.02$ & $1.181 \pm 0.042$ & 3.455 & This work \\
\hline $\mathrm{La}_{0.8} \mathrm{Ba}_{0.1} \mathrm{Ca}_{0.1} \mathrm{MnO}_{3}$ & CI & - & - & - & $3.341 \pm 0.003$ & This work \\
\hline Mean-field model & Theory & - & 0.5 & 1 & 3 & 57 \\
\hline 3D Ising model & Theory & - & $0.325 \pm 0.002$ & $1.241 \pm 0.002$ & $4.82 \pm 0.002$ & 57 \\
\hline Tri-critical mean-field model & Theory & - & 0.25 & 1 & 5.0 & 57 \\
\hline 3D Heisenberg model & Theory & - & $0.365 \pm 0.003$ & $1.336 \pm 0.004$ & $4.80 \pm 0.04$ & 57 \\
\hline $\mathrm{La}_{0.8} \mathrm{Sr}_{0.2} \mathrm{MnO}_{3}$ & - & 315 & $0.05 \pm 0.02$ & $1.08 \pm 0.03$ & $3.13 \pm 0.20$ & 58 \\
\hline $\mathrm{La}_{0.67} \mathrm{Ba}_{0.33} \mathrm{Mn}_{0.98} \mathrm{Ti}_{0.02} \mathrm{O}_{3}$ & - & 310 & $0.551 \pm 0.008$ & $1.020 \pm 0.024$ & 2.851 & 59 \\
\hline $\mathrm{La}_{0.7} \mathrm{Ca}_{0.3} \mathrm{Mn}_{0.95} \mathrm{Ti}_{0.05} \mathrm{O}_{3}$ & - & 136 & $0.601 \pm 0.02$ & $1.171 \pm 0.01$ & $2.95 \pm 0.01$ & 60 \\
\hline $\mathrm{La}_{0.7} \mathrm{Ca}_{0.05} \mathrm{Sr}_{0.25} \mathrm{MnO}_{3}$ & - & 344 & 0.42 & 1.14 & 3.7 & 61 \\
\hline $\mathrm{La}_{0.67} \mathrm{Ba}_{0.33} \mathrm{Mn}_{0.9} \mathrm{Cr}_{0.1} \mathrm{O}_{3}$ & - & 324 & $0.378 \pm 0.05$ & $1.357 \pm 0.002$ & 4.589 & 62 \\
\hline $\mathrm{La}_{0.67} \mathrm{Ba}_{0.33} \mathrm{MnO}_{3}$ & - & 306 & $0.356 \pm 0.004$ & $1.120 \pm 0.003$ & $4.15 \pm 0.05$ & 63 \\
\hline
\end{tabular}

Table 4 Comparison of critical exponents for our sample with various models; MAP, KF, Critical Isotherm (Cl) 
Table 5 Values of different parameters used to fit the experimental data to eqns (20) and (21)

\begin{tabular}{llllll}
\hline System & Region & $A^{\prime \prime}$ & $B$ & $\alpha$ and $\alpha^{\prime}$ & Ref. \\
\hline $\mathrm{La}_{0.8} \mathrm{Ba}_{0.1} \mathrm{Ca}_{0.1} \mathrm{MnO}_{3}$ & $T<T_{\mathrm{M}-\mathrm{SC}}$ & $0.00311 \pm 1.90479 \times 10^{-4}$ & $-9.92385 \pm 3.03029 \times 10^{-4}$ & 0.004 & This work \\
& $T>T_{\mathrm{M}-\mathrm{SC}}$ & $-0.00114 \pm 1.52525 \times 10^{-4}$ & $-0.00286 \pm 2.79734 \times 10^{-4}$ & 0.004 & This work
\end{tabular}

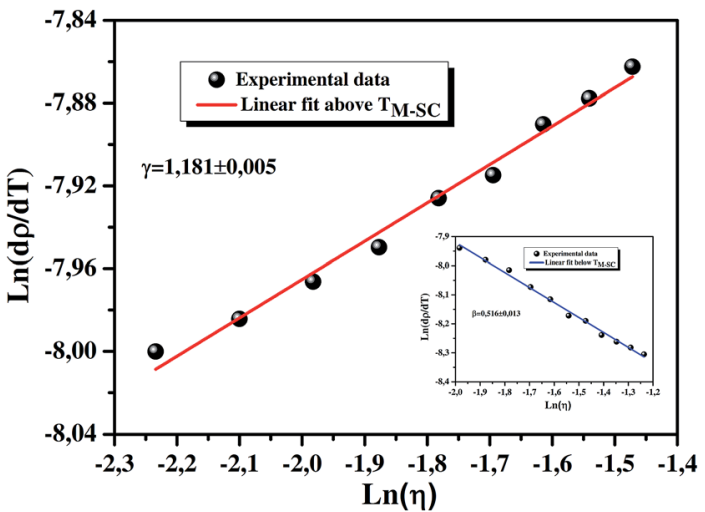

Fig. $11 \ln (\mathrm{d} \rho / \mathrm{d} T)$ vs. $\ln \left[\left(T-T_{M-s c}\right) / T_{M-s c}\right]$ above $T_{M-s c}$ under $5 T$. The inset shows $\ln (\mathrm{d} \rho / \mathrm{d} T)$ vs. $\ln \left[\left(T-T_{M-s C}\right) / T_{M-s C}\right]$ below $T_{M-s C}$ under $5 T$.

data is fitted by a straight line with a slope $1 / \delta$ using eqn (14). The value of $\delta$ is equal to $3.341 \pm 0.003$ in our case.

The critical exponents have to fulfill the Widom scaling relation: ${ }^{52,53}$

$$
\delta=1+\gamma / \beta
$$

Using the above determined values of exponents $\beta$ and $\gamma$, eqn (18) yields $\delta=3.344$ for $\beta, \gamma$ evaluated from MAP and $\delta=$ 3.455 for $\beta, \gamma$ obtained by the KF method. Thus, the critical exponents found in this study obey the Widom scaling relation remarkably well (Table 4).

3.4.2. Critical parameters determined from electrical resistivity. For a better understanding of the nature of phase transition, we tried to determine the values of the critical exponents close to second order metal-semiconductor transition and to assign one of these models to second order systems. According to the Fisher-Langer theory, ${ }^{54}$ specific heat at constant pressure $\left(C_{\mathrm{p}}\right)$ and at the phase transition temperature is proportional to the temperature derivative of the resistivity at $T=T_{\mathrm{M}-\mathrm{SC}}$. The thermal derivative of the resistivity is given by Fisher-Langer as:

$$
C_{\mathrm{p}} \propto(\mathrm{d} \rho / \mathrm{d} T)=(\mathrm{d} \rho / \mathrm{d} \eta)=\eta-\alpha
$$

here, $\alpha$ is the specific heat critical exponent and $\eta=\left(T-T_{\mathrm{M}-\mathrm{SC}}\right) /$ $T_{\mathrm{M}-\mathrm{SC}}$ is reduced temperature.

The two power law forms of eqn (19) below and above $T_{\mathrm{M}-\mathrm{SC}}$ given by Geldart et al..$^{55}$ are:

$$
\begin{aligned}
C(T) & =\left[1 / \rho\left(T_{\mathrm{C}}\right) \mathrm{d} \rho / \mathrm{d} T\right] \\
& =\frac{A^{\prime \prime}}{\alpha}\left[(-\eta)^{-\alpha}-1\right]+B^{+} \text {for } T<T_{\mathrm{M}-\mathrm{SC}} \\
C^{\prime}(T) & =\left[1 / \rho\left(T_{\mathrm{C}}\right) \mathrm{d} \rho / \mathrm{d} T\right] \\
& =\frac{A^{\prime \prime+}}{\alpha^{\prime}}\left[(-\eta)^{-\alpha}-1\right]+B^{-} \text {for } T>T_{\mathrm{M}-\mathrm{SC}}
\end{aligned}
$$

where $A^{\prime \prime}$ and $B$ are constants, $\alpha$ and $\alpha^{\prime}$ are specific heat critical exponents below and above $T_{\mathrm{M} \text {-sc }}$. The temperature derivative of resistivity normalized with respect to its value at $T_{\mathrm{M} \text {-SC, }}$, $\left[1 / \rho\left(T_{\mathrm{M}-\mathrm{SC}}\right) \mathrm{d} \rho / \mathrm{d} T\right]$ against $\eta$ is shown in Fig. 10 for our sample, and eqns (20) and (21) are fitted below and above $T_{\mathrm{M}-\mathrm{SC}}$ in the same figures. The solid lines passing through the data the are best fits in the two regions. The values of constants $A^{\prime \prime}, B$ and the specific heat critical exponents below and above $T_{\mathrm{M}-\mathrm{sC}}$ are obtained from the fitting analysis for the sample (seen in Table 5). Consequently, our model could be any one of the models such as mean field, 3D-Ising or tricritical mean-field.

Thus, it is somehow difficult to distinguish which one of them is the best for the determination of the critical exponents. Other critical exponents $\beta$ and $\gamma$ are calculated from the Suezaki-Mori model ${ }^{56}$ which relates the temperature derivative of the electrical resistivity to the reduced temperature $(\eta)$ magnetic ordering as follows:

$$
\begin{gathered}
{[\mathrm{d} \rho / \mathrm{d} T]=-B_{+} \eta^{-(\alpha+\gamma-1)} \text { for } T>T_{\mathrm{M}-\mathrm{SC}}} \\
{[\mathrm{d} \rho / \mathrm{d} T]=-B_{\mathrm{g}} \eta^{-(\alpha+\gamma) / 2}+\mathrm{B}_{-} \eta^{-(\alpha+\gamma-1)} \text { for } T<T_{\mathrm{M}-\mathrm{SC}}}
\end{gathered}
$$

where the constant $B_{+}$and $\mathrm{B}_{-}$incorporates term involving the zone boundary energy gap $B_{\mathrm{g}}$, as already denoted. Taking natural logarithm on both the sides, eqn (22) can be rewritten as:

Table 6 Estimated critical exponents for our sample and theoretical models

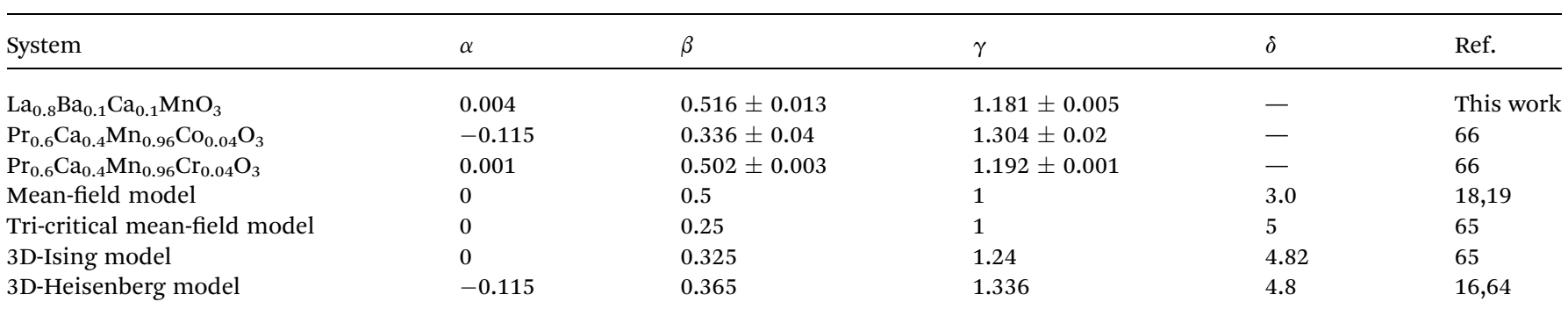




$$
\ln (\mathrm{d} \rho / \mathrm{d} T)=(\alpha+\gamma-1) \ln (\eta) \text { for } T>T_{\mathrm{M}-\mathrm{SC}}
$$

The slope of $\ln (\mathrm{d} \rho / \mathrm{d} T)$ versus $\ln (\eta)$ plot gives the value of $(\alpha+$ $\gamma-1$ ) (seen in Fig. 11). As $\alpha$ is obtained from the fit with FisherLanger method, we can obtain the value of $\gamma$. Substituting the value of a (derived from the figure in this relation), the critical exponent $\gamma$ was obtained as $\gamma=1.181 \pm 0.005$. The first term in eqn (23) involving $B_{\mathrm{g}}$ will be dominant at temperatures less than $T_{\mathrm{M} \text {-SC }}$ because the scaling law gives $(\alpha+\gamma) / 2-(\alpha+\gamma-1)=\beta$. As done earlier, eqn (23) can also be rewritten by taking natural logarithm on both sides as:

$$
\ln (\mathrm{d} \rho / \mathrm{d} T)=[(\alpha+\gamma) / 2-(\alpha+\gamma-1)] \ln (\eta) \text { for } T<T_{\mathrm{M}-\mathrm{SC}}
$$

Using scaling relation, $(\alpha+\gamma) / 2-(\alpha+\gamma-1)=\beta$ is equal to the value of critical exponent $\beta$. Hence, the direct slope of the plot $\ln (\mathrm{d} \rho / \mathrm{d} T)$ versus $\ln (\eta)$ below $T_{\mathrm{M} \text {-SC }}$ will give $\beta$ value. The inset of Fig. 11 presents $\ln (\mathrm{d} \rho / \mathrm{d} T)$ versus $\ln (\eta)$ below $T_{\mathrm{M}-\mathrm{SC}}$ for our sample, and the obtained value of slope is $0.516 \pm 0.013$. The critical exponents are determined in our case and listed in Table 6. Finally, one can see that Rushbrooke scaling relation $\alpha+2 \beta+\gamma=2$ (theory), which gives 2.217 for our sample is also satisfied. The estimated critical exponents from these experimental data and critical exponents from theoretical models such as the mean field, 3D Heisenberg, 3D-Ising and tricritical mean-field are listed in Table 6, and the estimated values for our sample are completely consistent with the mean field model.

\section{Conclusion}

The electrical properties of $\mathrm{La}_{0.8} \mathrm{Ba}_{0.1} \mathrm{Ca}_{0.1} \mathrm{MnO}_{3}$ have been studied. An important magnetoresistance at a $5 \mathrm{~T}$ applied magnetic field is achieved in the low-temperature region of our sample. Analyses of the electrical transport data found that SPH model is operative in the high temperature $\left(T>T_{\mathrm{M}-\mathrm{SC}}\right)$ regime in this manganite. The electrical conduction mechanism of low temperature $\left(T<T_{\mathrm{M}-\mathrm{SC}}\right)$ can be explained by a theory based on Kondo-like spin dependent scattering, e-e, e-m, and e-p scattering. Then, to study the transport mechanism in the entire range of temperature, we have used the theoretical percolation model, including the FM-metallic and PM-semiconductor region. Finally, we have examined the critical behavior of resistivity in $\mathrm{La}_{0.8} \mathrm{Ba}_{0.1} \mathrm{Ca}_{0.1} \mathrm{MnO}_{3}$ sample under different applied magnetic fields. The estimated critical exponents, obtained from resistivity, are $(\beta=0.516 \pm 0.013, \gamma=1.181 \pm 0.005$ and $\alpha=0.004)$. The values deduced from the critical exponents are close to the theoretical prediction of mean-field model. These results are in good agreement with the analysis of the critical exponents from magnetization measurements.

\section{References}

1 P. A. Yadav, A. V. Deshmukh, K. P. Adhi, B. B. Kale, N. Basavaih and S. I. Patil, Role of grain size on the magnetic properties of $\mathrm{La}_{0.7} \mathrm{Sr}_{0.3} \mathrm{MnO}_{3}$, J. Magn. Magn. Mater., 2013, 328, 86.
2 I. Mansuri and D. Varshney, Structure and electrical resistivity of $\mathrm{La}_{1-x} \mathrm{Ba}_{x} \mathrm{MnO}_{3}(0.25 \leq x \leq 0.35)$ perovskites, J. Alloys Compd., 2012, 513, 256.

3 M. D. Daivajna, A. Rao and G. S. Okram, Electrical, thermal and magnetic properties of $\mathrm{Bi}$ doped $\mathrm{La}_{0.7-x} \mathrm{Bi}_{x} \mathrm{Sr}_{0.3} \mathrm{MnO}_{3}$ manganites, J. Alloys Compd., 2014, 617, 345.

4 P. Zhang, P. Lampen, T. L. Phan, S. C. Yu, T. D. Thanh, N. H. Dan, V. D. Lam, H. Srikanth and M. H. Phan, Influence of magnetic field on critical behavior near a first order transition in optimally doped manganites: the case of $\mathrm{La}_{1-x} \mathrm{Ca}_{x} \mathrm{MnO}_{3}(0.2 \leq x \leq 0.4)$, J. Magn. Magn. Mater., 2013, 348, 146.

5 N. Sdiri, M. Bejar and E. Dhahri, The effect of the B-site size on the structural, magnetic and electrical properties of $\mathrm{La}_{0.7} \mathrm{Ca}_{0.3} \mathrm{MnO}_{3-\delta}$ compounds, J. Magn. Magn. Mater., 2007, 311, 512.

6 N. Kambhala and S. Angappane, Anisotropic magnetoresistance studies of polycrystalline $\mathrm{La}_{0.67} \mathrm{Ca}_{0.33} \mathrm{MnO}_{3}$, Phys. B, 2013, 411, 72.

7 R. Skini, A. Omri, M. Khlifi, E. Dhahri and E. K. Hlil, Large magnetocaloric effect in lanthanum-deficiency manganites $\mathrm{La}_{0.8-x} \square_{x} \mathrm{Ca}_{0.2} \mathrm{MnO}_{3}(0.00 \leq x \leq 0.20)$ with a first-order magnetic phase transition, J. Magn. Magn. Mater., 2014, 364, 5 .

8 M. Khlifi, M. Bejar, E. Dhahri, P. Lachkar and E. K. Hlil, Influence of Ca-deficiency on the magneto-transport properties in $\mathrm{La}_{0.8} \mathrm{Ca}_{0.2} \mathrm{MnO}_{3}$ perovskite and estimation of magnetic entropy change, J. Appl. Phys., 2012, 111, 103909.

9 M. S. Islam, D. T. Hanh, F. A. Khan, M. A. Hakim, D. L. Minh, N. N. Hoang, N. H. Hai and N. Chau, Giant magneto-caloric effect around room temperature at moderate low field variation in $\mathrm{La}_{0.7}\left(\mathrm{Ca}_{1-x} \mathrm{Sr}_{x}\right)_{0.3} \mathrm{MnO}_{3}$ perovskites, Phys. $B$, 2009, 404, 2495.

10 D. T. Hanh, M. S. Islam, F. A. Khan, D. L. Minh and N. Chau, Large magnetocaloric effect around room temperature in $\mathrm{La}_{0.7} \mathrm{Ca}{ }_{0.3-x} \mathrm{~Pb}_{x} \mathrm{MnO}_{3}$ perovskites, J. Magn. Magn. Mater., 2007, 310, 2826.

11 A. N. Ulyanov, D. S. Yang and S. C. Yu, Anomaly of local structure of $\mathrm{La}_{0.7} \mathrm{Ca}_{0.3-x} \mathrm{Ba}_{x} \mathrm{MnO}_{3}$ manganites at Curie temperature, J. Appl. Phys., 2003, 93, 7376.

12 M. H. Phan, S. B. Tian, S. C. Yu and A. N. Ulyanov, Magnetic and magnetocaloric properties of $\mathrm{La}_{0.7} \mathrm{Ca}{ }_{0.3-x} \mathrm{Ba}_{x} \mathrm{MnO}_{3}$ compounds, J. Magn. Magn. Mater., 2003, 256, 306.

13 R. Tripathi, V. P. S. Awana, N. Panwar, G. L. Bhalla, H. U. Habermier, S. K. Agarwal and H. Kishan, Enhanced room temperature coefficient of resistance and magnetoresistance of Ag-added $\mathrm{La}_{0.7} \mathrm{Ca}_{0.3-x} \mathrm{Ba}_{x} \mathrm{MnO}_{3}$ composites, J. Phys. D: Appl. Phys., 2009, 42, 17.

14 T. D. Thanh, T. H. Lee, T. L. Phan, D. A. Tuan and S. C. Yu, Influences of the first-to-second order magnetic phase transformation on the transport properties of $\mathrm{La}_{0.7} \mathrm{Ca}_{0.3-x} \mathrm{Ba}_{x} \mathrm{MnO}_{3}$ compounds, J. Appl. Phys., 2014, 115, $17 \mathrm{C} 706$.

15 U. L. Shinde, L. N. Singh and N. B. Srivastava, Small polaron transport and magnetoresistance in sol-gel prepared $\mathrm{Nd}_{0.7} \mathrm{Sr}_{0.3-x} \mathrm{Ca}_{x} \mathrm{MnO}_{3}(0 \leq x \leq 0.3)$ nanomanganite system, Phys. B, 2014, 452, 13. 
16 B. Padmanabhan, H. L. Bhat, S. Elizabeth, S. Rößler, U. K. Rößler, K. Dörr and K. H. Müller, Critical properties in single crystals of $\operatorname{Pr}_{1-x} \mathrm{~Pb}_{x} \mathrm{MnO}_{3}$, Phys. Rev. B: Condens. Matter Mater. Phys., 2007, 75, 024419.

17 S. Taran, B. K. Chaudhuri, S. Chatterjee, H. D. Yang, S. Neeleshwar and Y. Y. Chen, Critical exponents of the $\mathrm{La}_{0.7} \mathrm{Sr}_{0.3} \mathrm{MnO}_{3}, \quad \mathrm{La}_{0.7} \mathrm{Ca}_{0.3} \mathrm{MnO}_{3}$, and $\mathrm{Pr}_{0.7} \mathrm{Ca}_{0.3} \mathrm{MnO}_{3}$ systems showing correlation between transport and magnetic properties, J. Appl. Phys., 2005, 98, 8.

18 M. Ziese, Critical scaling and percolation in manganite films, J. Phys.: Condens. Matter, 2001, 13, 2919.

19 D. Kim, B. L. Zink, F. Hellman and J. M. D. Coey, Critical behavior of $\mathrm{La}_{0.75} \mathrm{Sr}_{0.25} \mathrm{MnO}_{3}$, Phys. Rev. B: Condens. Matter Mater. Phys., 2002, 65, 214424.

20 M. A. Gdaiem, S. Ghodhbane, A. Dhahri, J. Dhahri and E. K. Hlil, Effect of cobalt on structural, magnetic and magnetocaloric properties of $\mathrm{La}_{0.8} \mathrm{Ba}_{0.1} \mathrm{Ca}_{0.1} \mathrm{Mn}_{1-x} \mathrm{Co}_{x} \mathrm{O}_{3}(x$ $=0.00,0.05$ and 0.10) manganites, J. Alloys Compd., 2016, 681, 547.

21 G. Venkataiah, V. Prasad and P. Venugopal Reddy, Influence of A-site cation mismatch on structural, magnetic and electrical properties of lanthanum manganites, J. Alloys Compd., 2007, 429, 1.

$22 \mathrm{C}$. Zener, Interaction between the d-shells in the transition metals II. Ferromagnetic compounds of manganese with perovskite structure, Phys. Rev., 1951, 82, 403.

23 S. Hcini, S. Khadhraoui, S. Zemni, A. Triki, H. Rahmouni, M. Boudard and M. Oumezzine, Percolation model of the temperature dependence of resistivity in $\operatorname{Pr}_{0.67} \mathrm{~A}_{0.33} \mathrm{MnO}_{3}$ (A $=\mathrm{Ba}$ or Sr) manganites, J. Supercond. Novel Magn., 2013, 26, 2181.

24 S. Hcini, S. Zemni, A. Triki, H. Rahmouni and M. Boudard, Size mismatch, grain boundary and bandwidth effects on structural, magnetic and electrical properties of $\mathrm{Pr}_{0.67} \mathrm{Ba}_{0.33} \mathrm{MnO}_{3}$ and $\mathrm{Pr}_{0.67} \mathrm{Sr}_{0.33} \mathrm{MnO}_{3}$ perovskites, J. Alloys Compd., 2011, 509, 1394.

25 A. Urushibara, Y. Moritomo, T. Arima, A. Asamitsu, G. Kido and Y. Tokura, Insulator-metal transition and giant magnetoresistance in $\mathrm{La}_{1-x} \mathrm{Sr}_{x} \mathrm{MnO}_{3}$, Phys. Rev. B: Condens. Matter Mater. Phys., 1995, 51, 14103.

26 D. C. Worledge, G. J. Snyder, M. R. Beasley and T. H. Geballe, Anneal-tunable Curie temperature and transport of $\mathrm{La}_{0.67} \mathrm{Ca}_{0.33} \mathrm{MnO}_{3}$, J. Appl. Phys., 1996, 80, 5158.

27 M. Viret, L. Ranno and J. M. D. Coey, Magnetic localization in mixed-valence manganites, Phys. Rev. B: Condens. Matter Mater. Phys., 1997, 55, 8067.

28 G. J. Synder, R. Hiskes, S. DiCarolis, M. R. Beasley and T. H. Geballe, Intrinsic electrical transport and magnetic properties of $\mathrm{La}_{0.67} \mathrm{Ca}_{0.33} \mathrm{MnO}_{3}$ and $\mathrm{La}_{0.67} \mathrm{Sr}_{0.33} \mathrm{MnO}_{3}$ MOCVD thin films and bulk material, Phys. Rev. B: Condens. Matter Mater. Phys., 1996, 53, 14434.

29 G. Venkataiah and P. V. Reddy, Structural, magnetic and magnetotransport behavior of some Nd-based perovskite manganites, Solid State Commun., 2005, 136, 114.

$30 \mathrm{M}$. Ziese, Searching for quantum interference effects in $\mathrm{La}_{0.7} \mathrm{Ca}_{0.3} \mathrm{MnO}_{3}$ films on $\mathrm{SrTiO}_{3}$, Phys. Rev. B: Condens. Matter Mater. Phys., 2003, 68, 132411.
31 Y. Xu, J. Zhang, G. Cao, C. Jing and S. Cao, Low-temperature resistivity minimum and weak spin disorder of polycrystalline $\mathrm{La}_{2 / 3} \mathrm{Ca}_{1 / 3} \mathrm{MnO}_{3}$ in a magnetic field, Phys. Rev. B: Condens. Matter Mater. Phys., 2006, 73, 224410.

32 G. F. Dionne, Magnetic exchange and charge transfer in mixed-valence manganites and cuprates, J. Appl. Phys., 1996, 79, 5172.

33 N. F. Mott, Conduction in glasses containing transition metal ions, J. Non-Cryst. Solids, 1968, 1, 1.

34 B. S. Nagaraja, A. Rao and G. S. Okram, Structural, electrical, and colossal thermoelectric properties of $\mathrm{Dy}_{1-x} \mathrm{Sr}_{x} \mathrm{MnO}_{3}$ manganites, J. Supercond. Novel Magn., 2015, 28, 223.

35 S. Mollaha, H. L. Huang, H. D. Yang, S. Pal, S. Taran and B. K. Chaudhuri, Non-adiabatic small-polaron hopping conduction in $\mathrm{Pr}_{0.65} \mathrm{Ca}_{0.35-x} \mathrm{Sr}_{x} \mathrm{MnO}_{3}$ perovskites above the metal-insulator transition temperature, J. Magn. Magn. Mater., 2004, 284, 383.

36 J. B. Goodenough and J. S. Zhou, New forms of phase segregation, Nature, 1997, 386, 229.

37 G. Li, H. D. Zhou, S. J. Feng, X. J. Fan and X. G. Li, Competition between ferromagnetic metallic and paramagnetic insulating phases in manganites, J. Appl. Phys., 2002, 92, 1406.

38 M. Pattabiramana, G. Rangarajana and P. Murugaraj, Electrical conduction through bond percolation in $\mathrm{Nd}_{0.67} \mathrm{Sr}_{0.33} \mathrm{MnO}_{3}$, Solid State Commun., 2004, 132, 7.

39 N. Zaidi, S. Mnefgui, A. Dhahri, J. Dhahri and E. K. Hlil, Study of electrical transport and magnetoresistive properties of $\mathrm{La}_{0.67-x} \mathrm{Dy}_{x} \mathrm{~Pb}_{0.33} \mathrm{MnO}_{3}(x=0.00,0.10$ and 0.15), J. Alloys Compd., 2014, 616, 378.

$40 \mathrm{Y}$. Sun, W. Tong, X. Xu and Y. Zhang, Tuning colossal magnetoresistance response by $\mathrm{Cr}$ substitution in $\mathrm{La}_{0.67} \mathrm{Sr}_{0.33} \mathrm{MnO}_{3}$, Appl. Phys., 2001, 78, 5 .

41 F. Elleuch, M. Triki, M. Bekri, E. Dhahri and E. K. Hlil, A-site deficiency-dependent structural, magnetic and magnetoresistance properties in the $\operatorname{Pr}_{0.6} \mathrm{Sr}_{0.4} \mathrm{MnO}_{3}$ manganites, J. Alloys Compd., 2015, 620, 249.

42 N. Kallel, K. Frohlich, S. Pignard, M. Oumezzine and H. Vincent, Structure, magnetic and magnetoresistive properties of $\mathrm{La}_{0.7} \mathrm{Sr}_{0.3} \mathrm{Mn}_{1-x} \mathrm{Sn}_{x} \mathrm{O}_{3}$ samples $(0 \leq x \leq$ 0.20), J. Alloys Compd., 2005, 399, 20.

43 W. Cheikh-Rouhou Koubaa, M. Koubaa and A. Cheikhrouhou, Structural, magnetotransport, and magnetocaloric properties of $\mathrm{La}_{0.7} \mathrm{Sr}_{0.3-x} \mathrm{Ag}_{x} \mathrm{MnO}_{3}$ perovskite manganites, J. Alloys Compd., 2008, 453, 42.

44 N. Moutis, I. Panagiotopoulos, M. Pissas and D. Niarchos, Structural and magnetic properties of $\mathrm{La}_{0.67}\left(\mathrm{Ba}_{x} \mathrm{Ca}_{1-x}\right)_{0.33} \mathrm{MnO}_{3}$ perovskites $(0<\sim x<\sim 1)$, Phys. Rev. B: Condens. Matter Mater. Phys., 1999, 59, 1129.

45 X. Zhu, Y. Sun, X. Luo, H. Lei, B. Wang, W. Song, Z. Yang, J. Dai, D. Shi and S. Dou, Crossover of critical behavior in $\mathrm{La}_{0.7} \mathrm{Ca}_{0.3} \mathrm{Mn}_{1-x} \mathrm{Ti}_{x} \mathrm{O}_{3}$, J. Magn. Magn. Mater., 2010, 322, 242.

$46 \mathrm{~J}$. Yang and Y. P. Lee, Critical behavior in Ti-doped manganites $\mathrm{LaMn}_{1-x} \mathrm{Ti}_{x} \mathrm{O}_{3}(0.05 \leq x \leq 0.2)$, Appl. Phys. Lett., 2007, 91, 142512.

47 D. Kim, B. Revaz, B. L. Zink, F. Hellman, J. J. Rhyne and J. E. Mitchell, Tricritical point and the doping dependence 
of the order of the ferromagnetic phase transition of $\mathrm{La}_{1-x} \mathrm{Ca}_{x} \mathrm{MnO}_{3}$, Phys. Rev. Lett., 2002, 89, 227202.

48 A. Arrott and J. E. Noakes, Approximate equation of state for nickel near its critical temperature, Phys. Rev. Lett., 1967, 19, 786.

49 A. Schwartz, M. Scheffler and S. M. Anlage, Determination of the magnetization scaling exponent for single-crystal $\mathrm{La}_{0.8} \mathrm{Sr}_{0.2} \mathrm{MnO}_{3}$ by broadband microwave surface impedance measurements, Phys. Rev. B: Condens. Matter Mater. Phys., 2000, 61, R870.

50 J. S. Kouvel and M. E. Fisher, Detailed magnetic behavior of nickel near its curie point, Phys. Rev. B: Condens. Matter Mater. Phys., 1964, 136, A1626.

51 M. E. Fisher, S. K. Ma and B. G. Nickel, Critical exponents for long-range interactions, Phys. Rev. Lett., 1972, 29, 917.

52 B. Widom, Equation of state in the neighborhood of the critical point, J. Chem. Phys., 1965, 43, 3898.

53 B. Widom, Degree of the critical isotherm, J. Chem. Phys., 1964, 41, 1633.

54 M. E. Fisher and J. S. Langer, Resistive anomalies at magnetic critical points, Phys. Rev. Lett., 1968, 20, 665.

55 D. J. W. Geldart and T. G. Richard, Theory of spin-fluctuation resistivity near the critical point of ferromagnets, Phys. Rev. B: Condens. Matter Mater. Phys., 1975, 12, 5175.

56 Y. Suezaki and Y. Mori, Dynamic critical phenomena in magnetic systems II electrical resistivity near the néel point, Prog. Theor. Phys., 1969, 41, 1177.
57 H. E. Stanley, Introduction to phase transitions and critical phenomena, Oxford University Press, London, 1971.

58 C. V. Mohan, M. Seeger, H. Kronmüller, P. Murugaraj and J. Maier, J. Magn. Magn. Mater., 1998, 83, 348.

59 M. Oumezzine, O. Peña, S. Kallel and S. Zemni, Solid State Sci., 2011, 13, 1829.

60 A. Berger, G. Camopillo, P. Vivas, J. E. Pearson, S. D. Bader, E. Baca and P. Prieto, J. Appl. Phys., 2002, 91, 8393.

61 M. H. Phan, V. Franco, N. S. Bingham, H. Srikanth, N. H. Hur and S. C. Yu, J. Alloys Compd., 2010, 508, 238.

62 M. Oumezzine, O. Peña, S. Kallel and M. Oumezzine, J. Alloys Compd., 2012, 539, 116.

63 N. Mautis, I. Panagiotopoulos, M. Pissas and D. Niarchos, Phys. Rev. B: Condens. Matter Mater. Phys., 1999, 59, 1129.

64 S. Taran, B. K. Chaudhuri, S. Chatterjee, H. D. Yang, S. Neeleshwar and Y. Y. Chen, Critical exponents of the $\mathrm{La}_{0.7} \mathrm{Sr}_{0.3} \mathrm{MnO}_{3}, \quad \mathrm{La}_{0.7} \mathrm{Ca}_{0.3} \mathrm{MnO}_{3}, \quad$ and $\mathrm{Pr}_{0.7} \mathrm{Ca}_{0.3} \mathrm{MnO}_{3}$ systems showing correlation between transport and magnetic properties, J. Appl. Phys., 2005, 98, 103903.

65 D. Ginting, D. Nanto, Y. D. Zhang, S. C. Yu and T. L. Phan, Influences of Ni-doping on critical behaviors of $\mathrm{La}_{0.7} \mathrm{Sr}_{0.3} \mathrm{Mn}_{1-x} \mathrm{Ni}_{x} \mathrm{O}_{3}$, Phys. B, 2013, 421, 17.

66 R. Thiyagarajan, S. Esakki Muthu, G. Kalaiselvan and R. Mahendran Arumugam, Critical behavior of resistivity in the pressure-induced first to second order transition in $\mathrm{Pr}_{0.6} \mathrm{Ca}_{0.4} \mathrm{Mn}_{0.96} \mathrm{~B}_{0.04} \mathrm{O}_{3}$ ( $\mathrm{B}=\mathrm{Co}$ and $\mathrm{Cr}$ ), J. Alloys Compd., 2015, 618, 159-166. 